\title{
An Attempt to Predict Manoeuvring Indices Using AIS Data for Automatic OD Data Acquisition
}

\author{
Takeshige Nakano.* Kazuhiko Hasegawa.** \\ *Graduate School of Engineering, Osaka University, Osaka Japan \\ (Tel: +81-6-6877-5111,EXT: 3504; e-mail: takeshige_nakano@naoe.eng.osaka-u.ac.jp). \\ **Graduate School of Engineering, Osaka University, Osaka, Japan (e-mail: hase@naoe.eng.osaka-u.ac.jp)
}

\begin{abstract}
Marine traffic simulation system is a useful tool to access safety in congested waterways. In the simulation system, OD (Origin and Destination) data, ship type, dimension and manoeuvring indices $\mathrm{K}$ and $\mathrm{T}$ in Nomoto's equation are needed as input data. These input data can be created analyzing AIS data. However, in the present stage, manoeuvrability of ships has been roughly determined on the basis of ship type and length. This research is an attempt to predict them from AIS data. It will be useful for future data acquisition system for marine traffic simulation, autopilot setting or some statistical analysis.
\end{abstract}

Keywords: Ship manoeuvrability, Nonlinear programming, AIS, Prediction

\section{INTRODUCTION}

AIS(Automatic Identification System) is the system to share dynamic and static information such as ship velocity, position, ship type and ship length among ships which are sailing at close distance, and it has been introduced by IMO(International Maritime Organisation) since 2002. Recently, many researches have been developed regarding safety assessment of ship and aids for navigation by using ICT (Information Communication Technology), and AIS system is also used for these targets. However, since AIS is wireless connection between ships and land station, some data are untransmitted and reliability of this system is still disputable. Furthermore, non-availability of some data, for example, rudder angle, usage of AIS is limited in academic research. Hence, in this research, some missing or invalid data, such as heading angle and rate of turn are estimated, and by using these data, sailed rudder angle and manoeuvring indices $K$ and $T$ are predicted by optimisation method. These predicted data are important because normally, manoeuvring indices are only acquired from sea trial test such as zigzag test, but if it is predictable from AIS data, it can be used not only for marine traffic simulation, but it is also applicable for aids for navigation.

\section{AIS}

AIS is useful system that enables ships in close proximity to exchange their dynamic and static information automatically and periodically to support their safety navigation. It is also used to avoid collision, terrorism, piracy and to check ships around and cargo types of them as well. Various researches have been studied using this AIS system so far.
As mentioned above, there are two types of information in AIS, dynamic and static one. Component of dynamic information are received date and time, status (sailing, anchor), yaw rate, velocity, position and heading angle. On the other hand, IMO number, ship name, MMSI (Maritime Mobile Service Identity) type of ship, dimension, destination, ETA (Estimated Time Arrival) and current maximum draught are provided as static data.

\subsection{Data Intervals}

Dynamic AIS data are updated at specific time intervals depend on ship status (Hata, 2008). Meanwhile, static data is refreshed every 6 minutes or when information has changed. Time intervals for dynamic data are shown as Table 1.

Table 1. Data intervals of dynamic information

\begin{tabular}{|c|c|}
\hline Ship status & Interval \\
\hline $\begin{array}{c}\text { At anchor or moored and not } \\
\text { moving faster than } 3 \text { knots }\end{array}$ & $3(\mathrm{~min})$ \\
\hline $\begin{array}{c}\text { Ship at anchor or moored and } \\
\text { moving faster than 3 knots }\end{array}$ & $10(\mathrm{~s})$ \\
\hline Ship 0-14 knots & $10(\mathrm{~s})$ \\
\hline $\begin{array}{c}0-14 \text { knots and changing } \\
\text { course }\end{array}$ & $3-1 / 3(\mathrm{~s})$ \\
\hline Ship 14-23 knots & $6(\mathrm{~s})$ \\
\hline $\begin{array}{c}\text { Ships }>14 \text { knots and } \\
\text { changing course }\end{array}$ & $2(\mathrm{~s})$ \\
\hline
\end{tabular}

\subsection{Contents of AIS}




\subsection{Problem of AIS}

Reliability and lost data are the biggest difficulties of AIS. As mentioned above, because of wireless connection, many data are missing or not updated at appropriate intervals. Especially, rate of turn and rudder angle are essential data to predict manoeuvring indices in this research, but rudder angle is not included and most cases, rate of turn in AIS data only indicates positive or negative turn, because it is calculated by the difference of two previous values of heading angle. Therefore, rate of turn is predicted from heading angle. There are 3 steps in predicting rate of turn as explained below.

1. Heading angle of AIS is interpolated linearly into every 1 (s)

2. Differentiate numerically these heading angle

3. Calculate mean value of this calculated rate of turn in $10(\mathrm{~s})$

This is how rate of turn is predicted. Mean value of rate of turn is calculated in 10 (s) because it turned out to show reasonable result by try-and-error. The result of predicted rate of turn is compared to measured data to confirm the accuracy as shown in Fig.1.

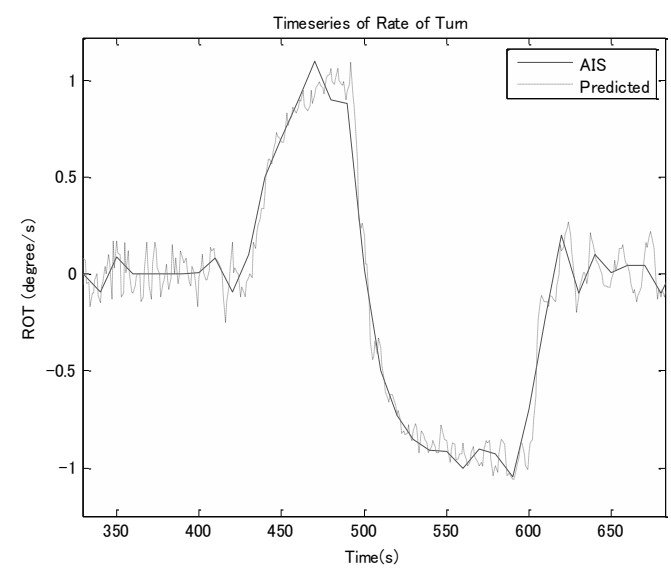

Fig.1. Comparison of rate of turn

In this figure, dot line represents calculated rate of turn and solid line is measured data by indicator. As you can clearly see in this figure, both lines are quite similar and our methodology for predicting rate of turn seems to be appropriate.

\section{OPTIMISATION METHOD}

Optimisation method is applied to predict manoeuvring indices and steered rudder angle. Method of optimisation is described in this chapter.

\subsection{Mathematical model for Ship Motion}

Nomoto's equation is applied to calculate ship motion because of its simplicity and computational speed. This model is seen as follows.
$T \dot{r}+r=K \delta$

Where $T$ : Time constant to the helm

$K$ : Index of turning ability

$\dot{r}$ : Angular acceleration

$r$ : Angular velocity

$\delta$ : Rudder angle

\subsection{Objective function}

Well known and widely used programming, MATLAB is used for all calculations in this paper. Installed function, called fmincon (Mathworks), based on quasi-newton method is utilised for optimisation. This function can minimize nonlinear objective function with constraints. There are two steps in optimisation. First one is to predict steered rudder angle by using appropriate initial value of $K$ and $T$. Next step is optimising $K$ and $T$ by the rudder angle that was optimised in the first step. In the first step, rudder angle is updated in each step and calculate heading angle and rate of turn from this refreshed rudder angle each time by above Nomoto's equation. Then, compare this calculated heading and rate of turn to measured, in this case, AIS data. Objective function is deference of these, and installed function finds best rudder angle that minimize this gap. Normalized value of heading angle and rate of turn is used to calculate differences between AIS data since dimension is different each other. In the second step, $K$ and $T$ is refreshed each step and calculate heading and rate of turn using this indices and optimised rudder angle from first step. And this difference is objective function for second step.

\subsection{Constraints}

Only rudder angle is constrained in this optimisation. It is set as \pm 20 (degree). We are recommended to use this value by ship sailor because they usually use \pm 20 (degree) as maximum rudder angle when they sail normally in any ship type and length. And \pm 30 (degree) or more is used only when the ship in emergency or velocity is very low.

\subsection{Initial value of $K$ and $T$}

In the first step of optimisation, initial value of $K$ and $T$ must be determined appropriately. In the second step where $K$ and $T$ are determined, modified optimised rudder angle from small one to large one is considered. This is because optimised rudder angle is strongly affected by initial value of $K$. Therefore, considering these varieties of rudder angle would help to make effect of initial value of $K$ smaller. Finally, many combinations of $K, T$ and rudder angle are acquired. In the matter of $T$, it doesn't disturb rudder angle, which means initial value of $T$ doesn't change after the optimisation. That's why it is very important how to determine initial value of $T$. It is fixed based on actual manoeuvrability of existing ships (Inoue, 2011). And its value can be seen in Table 2 . 
Table.2 Initial value of $T$

\begin{tabular}{|c|c|c|c|c|c|}
\hline Type & Length & $T(\mathrm{~s})$ & Type & Length & $T(\mathrm{~s})$ \\
\hline Cargo & $0-50$ & 20 & Passenger & $0-50$ & 10 \\
\hline & $50-100$ & 25 & & $50-100$ & 20 \\
\hline & $100-150$ & 35 & & $100-150$ & 30 \\
\hline & $150-200$ & 50 & & $150-200$ & 40 \\
\hline & $200-250$ & 60 & & $200-250$ & 50 \\
\hline & $250-300$ & 70 & & $250-300$ & 60 \\
\hline & $300-$ & 100 & & $300-$ & 80 \\
\hline Tanker & $0-50$ & 50 & Container & $0-50$ & 20 \\
\hline & $50-100$ & 80 & & $50-100$ & 25 \\
\hline & $100-150$ & 100 & & $100-150$ & 35 \\
\hline & $150-200$ & 150 & & $150-200$ & 45 \\
\hline & $200-250$ & 200 & & $200-250$ & 55 \\
\hline & $250-300$ & 230 & & $250-300$ & 65 \\
\hline & $300-$ & 250 & & $300-$ & 90 \\
\hline
\end{tabular}

\subsection{Determining $K$ and $T$}

After finishing two optimisation steps, several combinations of $K, T$ and rudder angle are acquired and one combination which is the most appropriate one needs to be determined. It is known that there are correlation between $K^{\prime}$ and $T^{\prime}$ (nondimensionalised values of $K$ and $T$ respectively) from past researches. Following figures are these examples. (Kansai Society of Naval Architects, Japan, 1984) In this figure, $C_{B}$ represents block coefficient and other coefficient will be explained later.

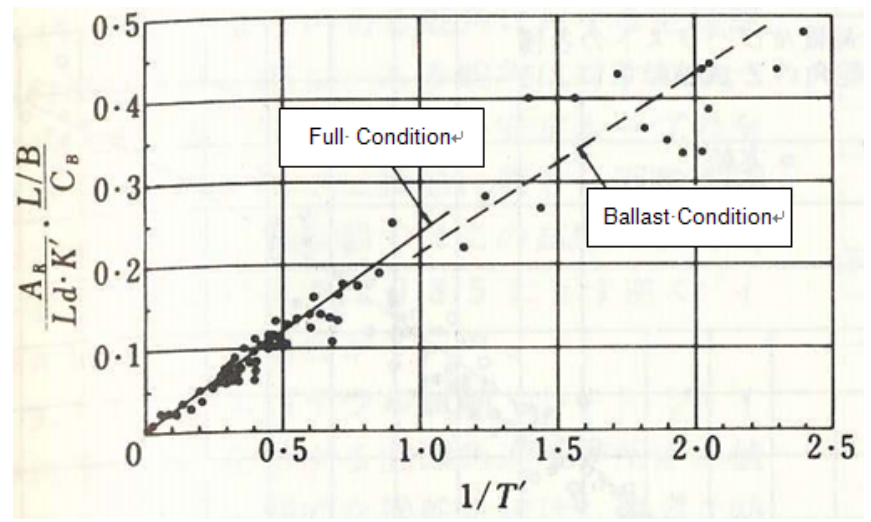

Fig.2 Correlation of $K^{\prime}$ and $T^{\prime}$ (Kansai Soceity of Naval Architects, Japan, 1984)

As you can see this figure, there are strong correlation between $K^{\prime}$ and $T$ ', and $K$ and $T$ is determined using this relationship finally. In this figure, area of rudder $A_{R}$ needs to be fixed. Since this value can't be found in AIS, the method to predict $A_{R}$ is introduced. It is decided by following equation. This is requirement of DNV (Kansai Society of Naval Architects, Japan, 1984).

$$
A_{R}=d L\left\{1+25(B / L)^{2}\right\} / 100
$$

Where

$d$ : Maximum draught

$L$ : Length over all

$B$ : Beam

Although above formulae has been utilised to estimate area of rudder in this paper, it turned out that this estimated value tends to be larger (safe side) than actual one. Following equation is newer method to estimate area of rudder and this has been utilised at DNV until $2009^{6}$. . This result would also be larger than actual size, however, this is more realistic and this relationship would be used in the future work.

There is another correlation(Kobayashi, 1978) between $K^{\prime}$ and $T$, and it is shown in Fig 3.

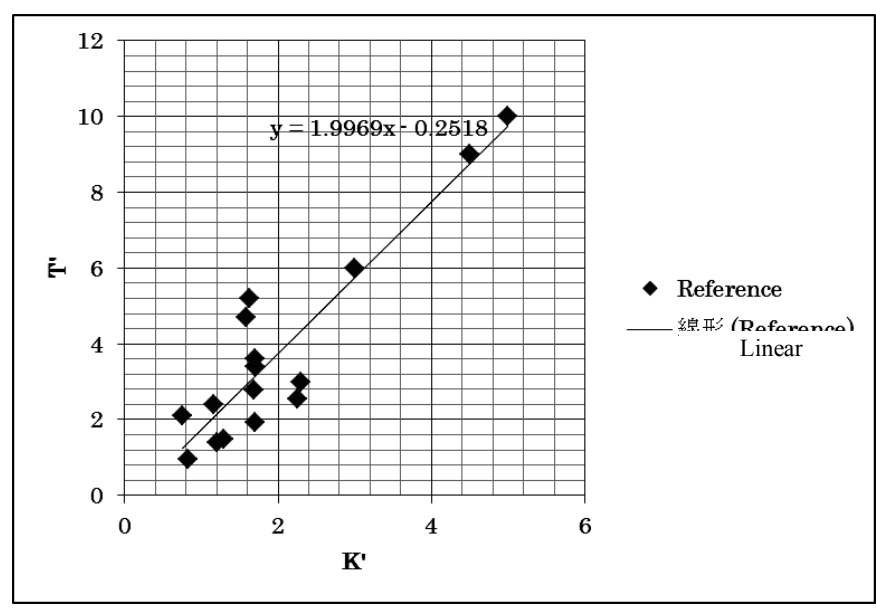

Fig. 3 Correlation of $K^{\prime}$ and $T$,

Each dot is plotted based on manoeuvrability of existing ships. Obviously, there are also interrelation that $T$, is twice bigger than $K^{\prime}$. Thus, $K$ and $T$ is determined depends on Fig 2 finally, and is able to confirm whether this result is suitable or not by this Fig 3.

\section{RESULTS}

\subsection{Results for a real ship}

To validate our proposed methodology, predicted rudder angle, and manoeuvrability is compared to navigational data of Fukae Maru, which is training ship of Kobe University equipped with yaw rate indicator. After validating our method, this approach is also applied to actual AIS data to calculate manoeuvring indices of arbitrary ship in the sea area. Firstly, some information regarding Fukaemaru is introduced. Following picture shows the overview of Fukae Maru. 


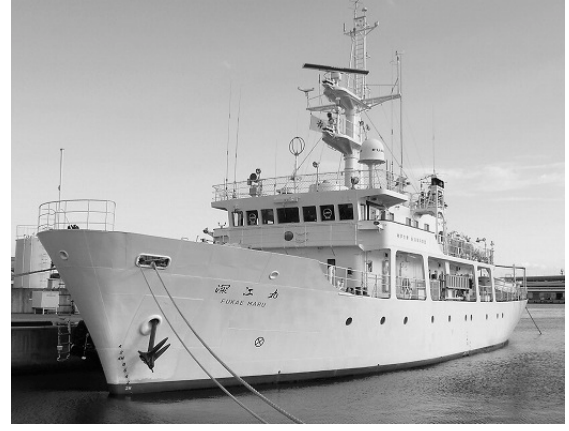

Fig.4 Overview of Fukae Maru

And Table. 3 shows the characteristics and Table.4 is the manoeuvrability of Fukae Maru.

Table.3 Characteristics and of Fukae Maru

\begin{tabular}{|c|c|}
\hline Type of ship & Training ship \\
\hline Date of completion & ${\text { Oct } 14^{\text {th }}, 1987}$ \\
\hline Length over all & $49.95(\mathrm{~m})$ \\
\hline Beam & $10.00(\mathrm{~m})$ \\
\hline Draught & $6.10 / 3.75(\mathrm{~m})$ \\
\hline Gross tonnage & $449(\mathrm{t})$ \\
\hline Displacement(Full $)$ & $776.5(\mathrm{t})$ \\
\hline Maximum velocity & $12.5(\mathrm{knot})$ \\
\hline Block coefficient & 0.53 \\
\hline
\end{tabular}

Table.4 Manoeuvrability of Fukae Maru

\begin{tabular}{|c|c|c|c|c|}
\hline $\begin{array}{c}\text { Experiment } \\
\text { condition }\end{array}$ & $T(\mathrm{~s})$ & $K^{\prime}$ & $T^{\prime}$ & Velocity(knot) \\
\hline 10 (degree) & 11.8 & 1.29 & 1.36 & 9.9 \\
\hline
\end{tabular}

These manoeuvring indices are calculated by using result of zigzag test. Initial value of $K$ and $T$ for optimisation is shown in Table 5.

Table.5 Conditions for optimisation

\begin{tabular}{|c|c|}
\hline Maximum rudder angle & $\pm 20($ degree $)$ \\
\hline Initial value of $\mathrm{K}$ & $0.1(1 / \mathrm{s})$ \\
\hline Initial value of $\mathrm{T}$ & $10(\mathrm{~s})$ \\
\hline
\end{tabular}

Optimisation is carried out in this condition. Initial value of $T$ is considered as 10(s) because block coefficiednt of Fukae Maru is the closest to that of passenger ship in four kind of ships in Table 2.

Fig. 5 shows the results for rudder angle. Solid line is actual sailed rudder angle from navigational data and dot line corresponds to optimised rudder angle.

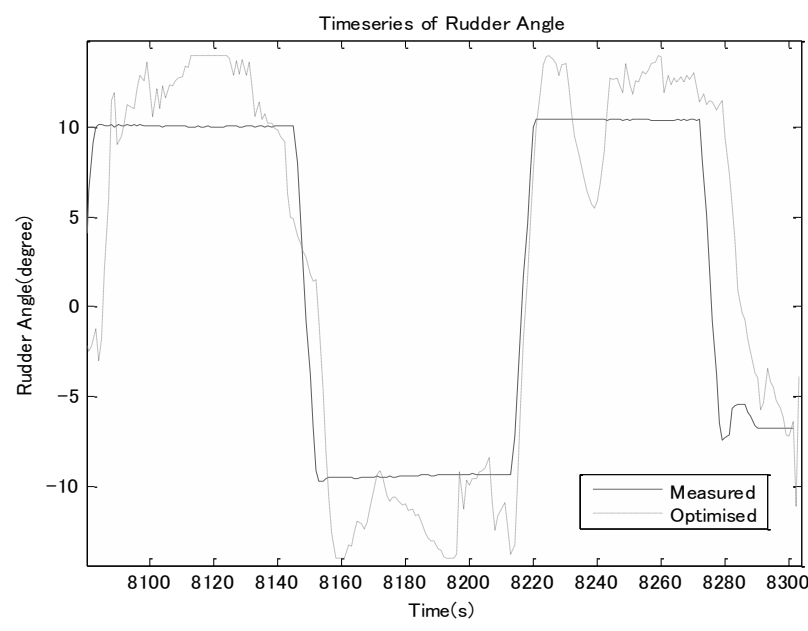

Fig.5 Comparison of rudder angle

As you can see, tendency of these two lines are similar and it seems that it is possible to use this objective function to estimate rudder angle.

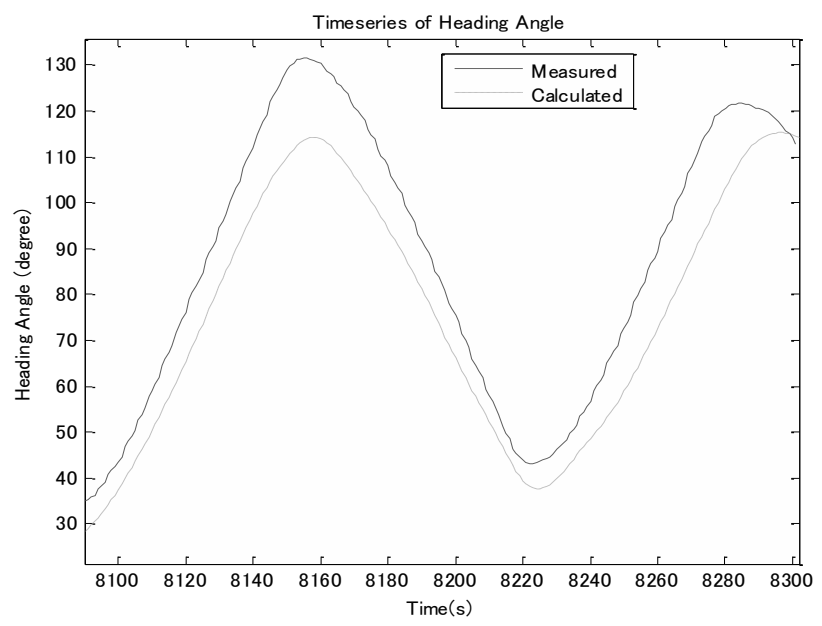

Fig.6 Comparison of heading angle

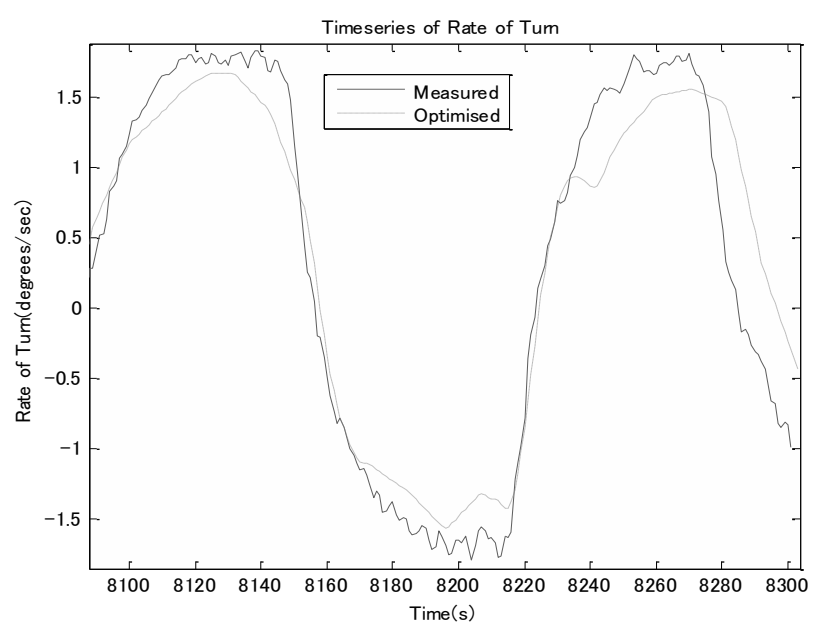

Fig.7 Comparison of rate of turn

In these two figures above, solid line is measured data and dot one is calculated value from optimised rudder angle. 
Manoeuvring indices from this optimisation can be seen below.

Table.6 Calculated manoeuvrability of Fukae Maru

\begin{tabular}{|c|c|c|c|c|}
\hline $\begin{array}{c}\text { Maximum } \\
\text { rudder angle }\end{array}$ & $\mathrm{T}(\mathrm{s})$ & $\mathrm{K}^{\prime}$ & $\mathrm{T}^{\prime}$ & Velocity(knot) \\
\hline 10 (degree) & 10 & 1.09 & 1.15 & 11.2 \\
\hline
\end{tabular}

Comparing this value to actual manoeuvrability on Table.4, this optimised result seems to be slightly smaller. This is because regarding $T$, it is affected by initial value of $T$ and this may cause small differences. Concerning about $K^{\prime}$, mean velocity is faster than experiment condition and this is the reason which makes differences. It is known that manoeuvring indices can be affected by the amount of rudder angle, or strength of turning motion. Fig.8 and Fig.9 shows the relationship between manoeuvring indices $K^{\prime}$ or $T^{\prime}$ and $r_{m}^{\prime}$. This $r_{m}^{\prime}$ represents average strength of turning motion and can be calculated as follows;

$$
\begin{aligned}
& \varphi_{m}=\sqrt{\sum_{i} \varphi^{2}\left(t_{i}\right) /(N+1)} \\
& r_{m}^{\prime}=\varphi_{m} \pi L /(180 U)
\end{aligned}
$$

Where, $\varphi$ : heading angle

$N$ : Total number of data

$L$ : Ship length

$U$ : Mean velocity

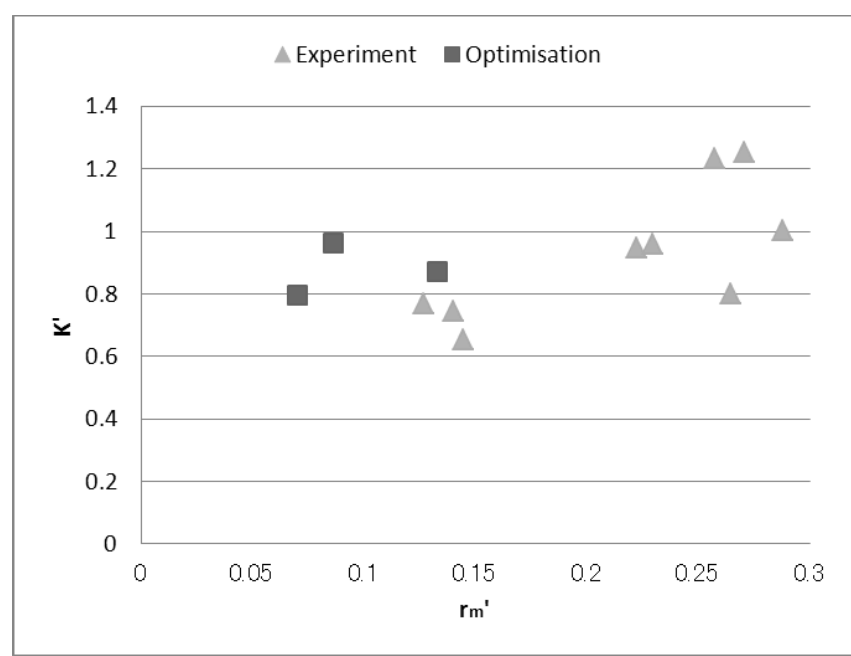

Fig. 8 Correlation of rm' and $K^{\prime}$

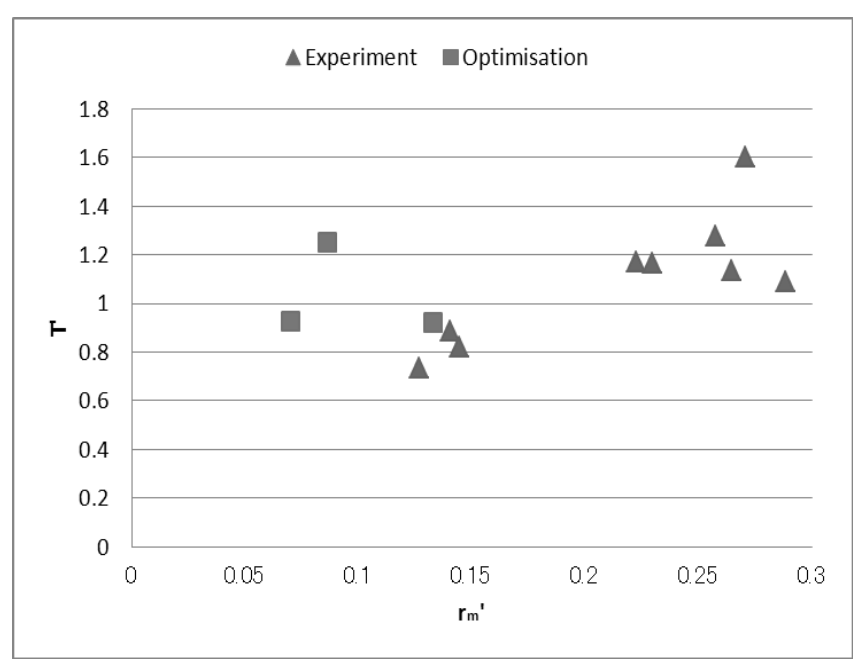

Fig.9 Correlation of $r_{m}$ and $T$,

In these figures, when turning motion is strong, which means rm' is big, optimisation result shows good agreement with experimental data. It could confirm that proposed method is appropriate to predict manoeuvring indices when ship is changing her course. This is because when ship keeps its course, yaw rate or heading angle is not affected by rudder angle but disturbunce is dominant, for example, wind, wave and tide.

\subsection{Results of arbitraly ships}

From the analysis of Fukaemaru, our method turned out to be good enough to predict manoeuvrability indices. Thus, it is also applied to actual AIS data. All the calculated manoeuvring indices are shown in Table 7.

Table 7. Calculated manoeuvring indices from AIS data

\begin{tabular}{|c|c|c|c|c|}
\hline MMSI & $K^{\prime}$ & $T$ & $K$ & $T$ \\
\hline Fukaemaru & 1.07 & 1.26 & 0.14 & 10 \\
\hline 431000331 & 0.99 & 2.27 & 0.056 & 40 \\
\hline 431000139 & 1.04 & 1.67 & 0.05 & 35 \\
\hline 306336000 & 0.85 & 1.78 & 0.044 & 35 \\
\hline 431000457 & 1.83 & 2.3 & 0.14 & 30 \\
\hline 431300324 & 1.11 & 2.13 & 0.059 & 40 \\
\hline 441129000 & 3.8 & 7.01 & 0.33 & 80 \\
\hline 636014868 & 3.11 & 6.18 & 0.084 & 230 \\
\hline 431500186 & 1.55 & 2.35 & 0.091 & 40 \\
\hline 440554000 & 3.12 & 6.56 & 0.26 & 80 \\
\hline 447001000 & 4.06 & 7.11 & 0.1445 & 200 \\
\hline
\end{tabular}

To confirm that calculation was carried out successfully, these results are plotted along with Fig 3. 


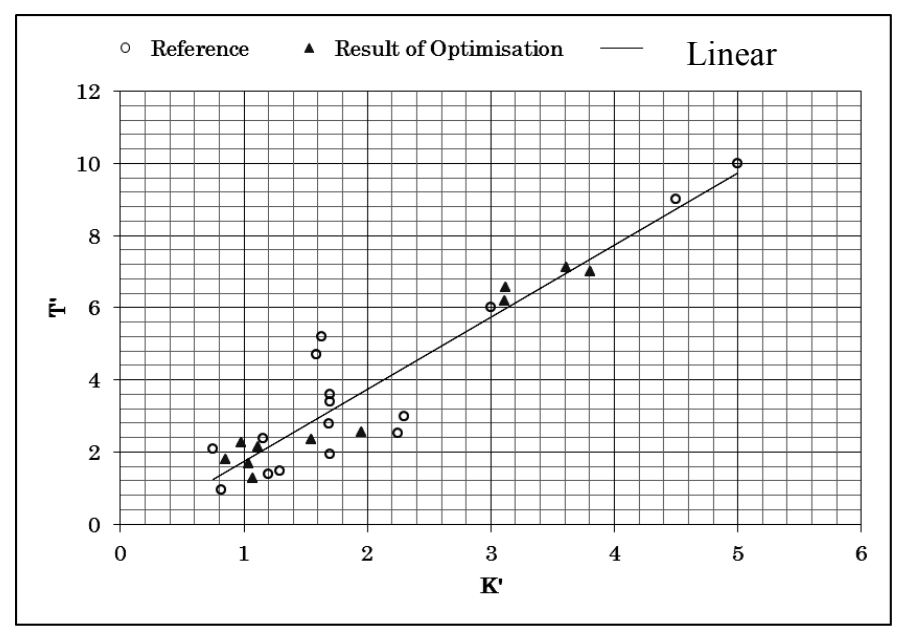

Fig. 10 Correlation between $K^{\prime}$ and $T^{\prime}$

In Fig 10, triangle shows the result of optimisation, and circle is manoeubrability of existing ships same as previous figure. It could be confirmed that each optimised results are close to approximate line and this means optimisation was carreid out successfully.

\section{CONCLUSION}

What is achieved in this paper are follows;

- $\quad$ The method to predict manoeuvrability indices $K$ and $T$ by using AIS is proposed.

- $\quad$ Non-linear programming is applied to optimise $K$ and $T$.

- $\quad$ By the experiment of Fukaemaru and relationship of $K^{\prime}$ and $T^{\prime}$, proposed method is validated successfully.

From Fig. 8 and Fig.9, it concluded that it is possible to predict appropriate manoeuvring indices only by using data which ship is changing her course, although these data are not always available. Hence, to develop some automatic system to find appropriate data will be one of our future tasks. Meanwhile, our final goal of this research is to get manoeuvring indices of arbitrary ships in close proximity only by AIS to create OD data automatically, to do so, calculation time needs to be much smaller. At this moment, it takes 1 to 4 hours for each ship. Furthermore input data for this calculation should be got automatically. Currently, we have to find some good data, when ship is changing her courses, by ourselves and it takes so much time. Some system to find useful data automatically should be invented, in this sense. These are our future tasks.

\section{REFERENCES}

K.Hata. (2008). Assessment of AIS Communication Traffic Volume in Congested Sea Area, Doctor thesis of Osaka University, Japan

K.Inoue, (2011). Theory and practice of ship handling, Seisanndo, Japan

E.Kobayashi. (1978). Prediction of Manoeuvring Indices by Optimisation Method, Master thesis of Osaka University, Japan
Handbook for Designing Ships ver.4, (1984). The Kansai Society of Naval Architects, Japan

\section{Mathworks,fmincon,}

http://www.mathworks.co.jp/help/toolbox/optim/ug/fmincon. $\underline{\mathrm{html}}$ 